\title{
A Study on the Trend of Web2.0 Research
}

\author{
Tao Zhang ${ }^{1}$ Kun Zhao ${ }^{2}$ Zhangxiang $\mathrm{Zhu}^{3}$ \\ ${ }^{1,3}$ Information College, Yunnan University of Finance and Economics, Kunming, China \\ ${ }^{2}$ Modern Education Technology Center, Yunnan University of Finance and Economics, \\ Kunming, China \\ Email: zhangtaozjt-006@163.com
}

\begin{abstract}
This paper uses CNKI as search source, and uses 'Web2.0' as the key word to search the research papers related to Web2.0 during the period of 2005 2009 in China. By using Bibliometric methods, this paper analyzes the overall situation, existing problem, solution strategies and trends of the domestic research on Web2.0 in recent years from the aspects such as age distribution of research literatures, hot topics, working journals, research institutions and core researchers, etc.
\end{abstract}

Keywords: Web2.0, Bibliometric Analysis, Analysis of current situation, Trends

\section{Introduction}

Web 2.0 was proposed in a brainstorming meeting of O'Reilly Media, Inc. and MediaLive, Inc. in March 2004. According to the definition given by O'Reilly, who is the proposer of the concept, Web2.0 is a network platform which across all Internet equipment ${ }^{[1]}$. Relative to Web1.0, Web2.0 is a new general term for Internet applications. The most important feature of Web2.0 includes personalization and decentralization. Web2.0 puts extremely importance on socialization, opening, sharing, participation and creation in Internet application.

Web2.0 has brought a real Internet revolution in which reading and writing coexist, transmission model is personalized, creation method is standardized, joint approach socialization, experience way facilitation and media approach intensification $^{[2]}$. Web2.0 services and applications have become a new area of network marketing, and its idea has begun to affect the management of enterprise, Web2.0 has cut the high cost of traditional enterprise to install, configure, maintain and upgrade the necessary software. Web2.0 concept such as Blog, SNS (Social Network Service), podcast, wiki, RSS(Really Simple Syndication) and more service will appear in the enterprise applications to improve the relationships between the company and potential and existing customers and give them the double identity of customer and partner. Web2.0 also has changed the production, using, sharing and manipulation mode of information. Some services of Web2.0 such as music, video, mashup-style applications, community have been applied by a number of network user ${ }^{[3]}$. Meanwhile, Both web2.0 applications in education and library and far-reaching implications web2.0 brings are more worthy researching and discussing ${ }^{[4][5]}$. Web2.0 is turning into an important application tools and academic focus.

China follows closely international pace on web2.0 research ${ }^{[6]}$ and domestic emerging research can be described as full swing. In order to completely understand the domestic development and research status of Web2.0, this article uses Bibliometrics to analyze the papers related to Web2.0 in the CNKI from 2005 to 2009 , revealing the research track and 
hotspot of Web2.0, analyzing the existing problems and proposing solving strategy from the study on the age distribution, theme distribution, hot research spots distribution, periodical distribution and so on.

\section{Materials and Methods}

\subsection{Materials}

This paper selects CNKI as literature sources. CNKI, which is the world's largest continuous dynamic updated database and provides researchers with highly integrated data, collects the full text of literature of 7451 kinds of journals and the rate of being embodied by CNKI for the papers exceeds $99 \%$. To this end, the database can obtain more comprehensive and effective literatures related to web2.0 and the results obtained can reflect present progress and trends of domestic researches on web2.0.

\subsection{Methods}

This paper uses 'web2.0' as the search strategy for searching into the CNKI database in this research, and search approach is 'Key words or title', the time range of published literature is ' 2005 2009', match model is 'Accurate', and other qualifications is default Database settings. First of all, a total of 948 papers were retrieved by means of the key words 'Web2.0' search, and then 555 papers were filtered out from 948 papers via the title 'Web2.0' search. Finally, through referring to abstracts or full text to remove these papers which are not associated with Web2.0, 549 papers were retained which were inserted into the SPSS .The reason of selecting the SPSS is based on the functions of its sorting, filtering and statistics.

\subsection{Bibliometric Methods}

Making bibliometric analysis ${ }^{[7]}$ on the age distribution, subject distribution, the hot research topics distribution and journal distribution and so on, so as to explore the research track and hotspot of Web2.0, analyze the existing problems, draw appropriate conclusions and predict the trends of the domestic research.

\section{Bibliometric Analysis}

\subsection{Distribution of age, theme and re- search focus}

There have 549 research literatures related to Web2.0 from 2005 to 2009 and the specific age distribution shows in table 1. From 2005 to 2006, the number of literatures is relatively small and totals 35 accounting for $6.4 \%$ of the total literatures; 96 literatures are published in 2007 and there has an increase of $210 \%$ compared to 2006; The number becomes double in 2008; In 2009, there has an increase of $16.6 \%$ compared to 2008 .

\begin{tabular}{|c|c|c|c|c|c|}
\hline Years & 2005 & 2006 & 2007 & 2008 & 2009 \\
\hline Papers & 4 & 31 & 96 & 193 & 225 \\
\hline Percentage & 0.7 & 5.7 & 17.5 & 35.2 & 40.9 \\
\hline Growth rate & 0 & 675 & 210 & 101 & 16.6 \\
\hline
\end{tabular}

Table 1: Distribution of number of literatures.

This article divides the research topics of Web2.0 into 8 groups through extracting thesis and Chinese abstract of the literatures ${ }^{[8][9][10][11]}$ : review, theoretical research, technology system research, analysis of impact(The former four belong to theoretical research ), application research in education, library, E-commerce, enterprise informatization, network marketing communication and other aspects(The last six are classified as application research).

Overview describes concept, ideas, characteristics, background and development process of Web2.0 and relations with web1.0 as well; Main theoretical research is that researches on 'Six Degrees of Separation', copyleft theory, long tail theory of Web2.0; Technology system 
research makes research on technology thought of Web2.0, basic technology (XML, Ajax, open API, PtoP transmission technology, etc.), application technology (Blog, Wiki, Tag, SNS, Rss, etc) and application of business models (Wiki, Tag, SNS, Rss, etc.); Analysis of impact points at far-reaching impact that Web2.0 brings into a particular field. Topic distribution of literatures related to Web2.0 from 2005 to 2009 shows in table 2.

Table 2 shows that research in 2005 focus on theoretical research and application research is few. In 2006, researches focus on overview and technical system which account respectively for $58.1 \%$ and $45.2 \%$ of the total of the year. In 2007 , researches on overview, technical system, application in education and library account respectively for $21.9 \%, 50 \%, 4 \%$ and $18.8 \%$. In 2008 , technology system research is still the hot spot, in addition, theoretical research keeps pace with application research. In 2009, application research surpasses theoretical research and the application in education, library, enterprise information and network marketing communication develops rapidly.

\begin{tabular}{|c|c|c|c|c|c|c|c|c|c|c|c|c|c|}
\hline \multirow{2}{*}{ 蛋 } & \multicolumn{5}{|c|}{ Theoretical research } & \multicolumn{7}{|c|}{ Application research } & \multirow[b]{2}{*}{$\stackrel{\overrightarrow{0}}{\stackrel{\vec{D}}{\Xi}}$} \\
\hline & 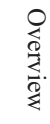 & 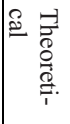 & 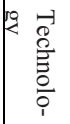 & $\begin{array}{l}\text { 芯 } \\
\text { ڤ̆ }\end{array}$ & 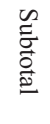 & 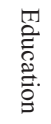 & 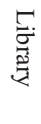 & 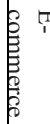 & 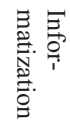 & 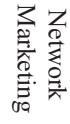 & $\begin{array}{l}\stackrel{0}{\vec{f}} \\
\stackrel{0}{0} \\
\dot{\omega}\end{array}$ & 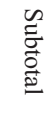 & \\
\hline 2005 & 2 & 2 & 3 & 2 & 9 & 0 & 1 & 1 & 0 & 0 & 1 & 3 & 4 \\
\hline 2006 & 18 & 6 & 14 & 10 & 48 & 2 & 6 & 3 & 3 & 6 & 1 & 21 & 31 \\
\hline 2007 & 21 & 11 & 48 & 11 & 91 & 23 & 18 & 4 & 6 & 9 & 8 & 68 & 96 \\
\hline 2008 & 50 & 23 & 71 & 34 & 178 & 36 & 48 & 10 & 20 & 13 & 35 & 162 & 193 \\
\hline 2009 & 36 & 14 & 82 & 31 & 163 & 49 & 76 & 7 & 26 & 17 & 38 & 213 & 225 \\
\hline Total & 127 & 56 & 218 & 88 & 489 & 110 & 49 & 25 & 55 & 45 & 83 & 467 & 549 \\
\hline
\end{tabular}

Table 2: Distribution of research topics.

\subsection{Distribution of author, working journal and research institution}

In order to make a comprehensive study of distribution and continuity of research, this article will count the number of the first author, second author and third author as a whole. Figure 1 shows that a total of 758 authors make different levels research on Web2.0 and publish literatures in the core journals from 2005 to 2009. The number of the authors issuing only one literature is 700 and accounts for $92.3 \%$ of the total; 50 authors issue respectively two literatures and account for $6.6 \% ; 6$ authors issue respectively three literatures and account for $0.8 \%$; The number of the authors issuing more than three literatures is 2 and accounts for $0.3 \%$, among the authors Wang Weijun publishes 7 papers and Gu Liping publishes 5. If regard someone publishes above 3 (including 3 ) literatures as core author, there have 8 core authors accounting for $1.1 \%$. Meanwhile, all core authors publish 31 literatures accounting for $5.6 \%$.

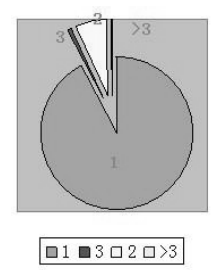

Fig. 1: Distribution of core author.

A total of 192 kinds of journals publish related papers. Among of them, 14 kinds of journals publish respectively more than 10 (including 10) articles accounting for $7.3 \%$ and the journals publish over 208 articles accounting for $37.9 \%$ in all. According to the discrete law of literature, 
academic literatures related to a subject will heavily concentrated in a small number of publications and the remaining small number of literatures are scattered in more publications. Figure 2 points out that the journals publishing more than 11 papers issue a total of 158 literatures accounting for $28.8 \%$ in all and also indicates that 'Modern Information'(MI), 'Tech information development \& economy'(TI), 'Software Guide'(SG), 'Library and information service'(LI), 'Scientific information'(SI), 'Information Science'(IS), 'Knowledge of Library and Information Science'(KL), 'Chinese audio-visual education'(CA) and 'Information and Documentation Services'(ID) are the major academic positions of Web2.0 research. The other 183 kinds of journals publish 391 papers.

Figure 3 shows that a total of 436 different departments, libraries and other research institutions ( $95 \%$ from universities) make research on Web2.0. The 8 institutions publishing more than 7 (including 7) papers publish in all 98 articles accounting for $17.9 \%$, which are Wuhan University(WH), Central China Normal University $(\mathrm{CN})$, Chinese Academy of Science(AS), East China Normal University(EN), Southwest University(SW),Zhon- gshan University(ZS), Shanghai University(SH), Nanjing University(NJ).

\subsection{Geographic and fund distribution}

According to the geographic distribution of literature, literatures related to Web2.0 distribute in 29 provinces and cities, specific data as shown in table 3 .

The number of literatures distributing in the eight provinces lie in east-central China is 335 accounting for $61.1 \%$. However, Only 65 literatures accounting for $13.7 \%$ distribute in the western provinces.

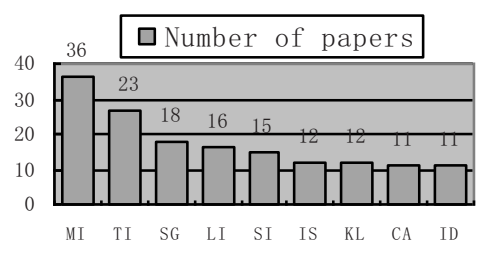

Fig. 2: Distribution of journal papers.

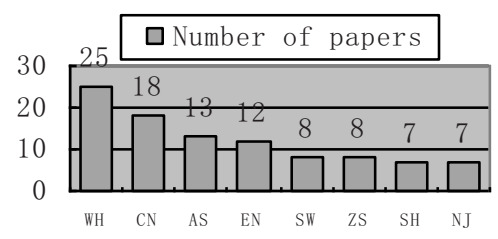

Fig. 3: Distribution of research institute.

This paper counts the number of national and provincial funds but the college funds. As shown in table 3, a total of 77 literatures with fund account for $14.1 \%$, there aren't funded research in 2005, the yearly proportion of literatures with fund keeps at about $10 \%$ from 2006 to 2008 and it soars to $19.1 \%$ in 2009 .

\section{The Conclusion and Comment of Web2.0 Study Based on the Analy- sis Results}

\subsection{Research status and trend}

From the above analysis we can draw several conclusions as follows.

(1) Research on Web2.0 has gone through 5 different stages and will go to its $6^{\text {th }}$ research stage. From the distribution of age, topic and hot spot we see something as follows. First, the concept of Web2.0 just entered into the domestic in 2005 and only individual scholars touched with new technology and new ideas, and most of researches focused on theoretical research. Second, Web2.0 concept was widely understood in 2006 and Web2.0 theory obtained a rapid development, the research of Web2.0 ap- 
plied to library and network marketing communication started. Third, theoretical studies made a great breakthrough in 2007, especially in the research of technical system which laid a solid foundation for wide application of Web2.0, then a rapid development has been achieved on the research on education, library, network marketing, e-commerce and enterprise information, moreover China ushered in the first year of marketing in this year. Fourth, new technology and concept have been comprehensive study and widely applied in 2008, theory research achieving more overall development and application research making a breakthrough in individual fields went hand in hand. Finally, based on the current Web- 2.0 ideas and technology, theory tended to be perfect and application research on the individual field have obtained All-round development in 2009. According to the law of longitudinal development in the past few years, applica- tion research will become future research mainstream tendency in the next few years and gain a breakthrough in more fields. In the meantime theory should advance with the times and achieve creative results. In summary, according to above analysis, this article will divide longitudinal develop progress into six stages of development.

Figure 4 intuitively shows the different six stages of research progress of theory and application research. On the whole, theory research is a stage (one year) earlier than application research in research progress which illustrates that the theory research comes first at home and is the theoretical basis and guide of application research. Therefore, the domestic research should strengthen theory research especially in the theoretical innovation in the future so as to build a solid theoretical foundation for the healthy and stable development of application research.

\begin{tabular}{|c|c|c|c|c|c|c|c|c|}
\hline Province & Hubei & Beijing & Guangdong & Shanghai & Jiangsu & Henan & Zhejiang & Hunan \\
\hline Papers & 66 & 58 & 50 & 44 & 41 & 28 & 25 & 23 \\
\hline Percentage & 12 & 10.6 & 9.1 & 8 & 7.5 & 5.1 & 4.6 & 4.2 \\
\hline
\end{tabular}

Table 3: Geographical distribution of literature.

(2) The number of papers related to Web2.0 shows the theoretical level and development progress of Web2.0 research. From the age distribution of literatures, the domestic research started at 2005 and relatively later than foreign study. Before 2007, Web2.0 was just the capital investment point of Internet companies and didn't bring these companies huge profits so that it didn't attract enough attention and acceptance from users ${ }^{[12]}$; However, because of considerable profits Web2.0 brought to certain business after 2007, domestic Web2.0 research developed rapidly and maintained a trend of rapid progression, on this account it gradually become the focus of attention ${ }^{[6]}$. From this we can say that the initial starting stage of
Web2.0 study is from 2005 to 2006 and the period from 2007 to 2009 can be regarded as the rapid development stage of Web2.0 research.

\begin{tabular}{|c|c|c|c|c|c|}
\hline Years & 2005 & 2006 & 2007 & 2008 & 2009 \\
\hline Number & 0 & 3 & 10 & 21 & 43 \\
\hline Percentage & 0 & 9.6 & 10.4 & 10.9 & 19.1 \\
\hline
\end{tabular}

Table 4: Distribution of literatures with fund.

(3) Web2.0 research has become an academic focus. In the journal distribution, the concern is that 36 papers published in 'Modern Information' account for 6.6\% of the total papers, which illustrates that the journal takes an advantage position in 
Web2.0 research; Meanwhile, in the top nine journals based on the number of published articles, 'Modern Information', 'Library and information service', 'Information Science', 'Knowledge of Library and Information Science', 'Chinese audio-visual education' and 'Information and Documentation Services' are all collected into CSSCI.
(4) University library plays an important role in Web2.0 research. The distribution of research institutions shows that university is the main force of Web2.0 research. What is noteworthy is that the number of university library reaches 148 accounting for $33.9 \%$ of the total institutions and the papers from university library account for $28.3 \%$ of the total.

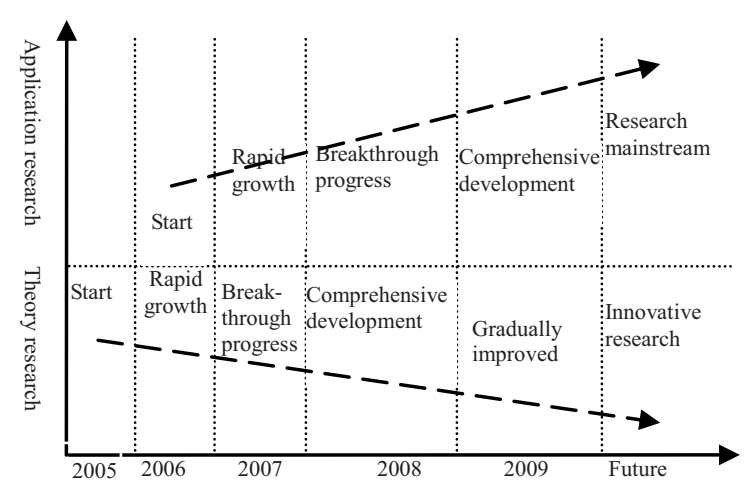

Fig. 4: Analysis diagram for Stage of development.

(5) The number of literatures with fund increases year by year and researches are centered largely in east-central china. According to the fund and geographic distribution of literature, we can see that the proportion of the literatures with fund increases year by year and reaches about $20 \%$ of the total in 2009 which indicates that Web2.0 research has always been the focus of attention and will attract more and more attention. Furthermore, we can realize that domestic Web2.0 research is focused in east-central china.

\subsection{The analysis of Existing Problems}

Although starting pretty late, the domestic Web2.0 research has been developed rapidly which closely followed the steps of overseas study in recent years. Meanwhile, many problems summarized based on the above analysis as follows:

(1) Lack of continuity and call for indeep research
The author distribution shows that only 8 authors accounting for $1.1 \%$ respectively published at least 3 articles. Among of them, Wang Weijun published 7 articles, $\mathrm{Gu}$ Liping published 5 and the remaining 5 authors respectively published 3 . Thus, domestic scholars have not formed a relatively active research groups and the continuity of the research needs to be strengthened. To most of scholars, there is only the preliminary and cognitive research but the late-stage continued and in-depth study, which makes it difficult for scholars to become the core author and achieve a breakthrough achievement. Given this, domestic authors need more perseverance and should make comprehensive analysis and in-depth research in the fields related to Web2.0 so as to bring a far-reaching impact to related fields and achieve great academic achievements.

(2) Lack of cooperation among research institutions

According to the distribution of re- 
search institutions and the geographic distribution, $95 \%$ of research institutions are from universities and scattered, and the number of papers is relatively large, however, the concentration degree is low, which results in the scattered distribution of scholars and the isolated study without teamwork. Considering that, scholars and research institutions should learn to share resource and cultivate the team cooperation spirit when carrying out their studies, so as to form a regional and authoritative research of Web2.0.

(3) Limiting research scope and overlapping study on some topics

Domestic Web2.0 research mainly focuses on the technical system and the application in education and library, the papers related to the above two aspects account for $75 \%$ of the total. The amount of papers is large and research topics are limited in a little scope, which results in repetitive study. For this reason, domestic scholars should strengthen communication with each other to avoid repetitive research and should deepen and broaden the research of Web2.0 to achieve comprehensive progress.

(4) Unbalanced development of domestic research

Geographic distribution of literature shows that 29 provinces and cities had published the relevant literature and the western provinces published a total of 65 articles accounting for only $13.7 \%$. We can see that the West China falls far behind the east-central in the research progress which is closely linked to regional economy development. We also know the application in enterprise information, E-commerce and Network marketing is relatively small and didn't attract enough attention in western regions. To this end, the western region should accelerate and enhance the research on the theory of Web2.0 so as to promote the development of application research and reduce the gap.

\section{Acknowledgement}

The work was supported by the Key Project of Science Research Program, Supported by Chinese Ministry of Education (208128).

\section{References}

[1] Tim O'Reilly, "What Is Web 2.0", O'Reily Network.Retrieved.2006

[2] Yue Zheng, "Status and Trend of China Web2.0",Economics and Management school of Beijing University of Posts and Telecommunications, 2006.

[3] Wei Wang, "Simple analysis for the application and development trend of Web2.0",China Science and Technology Information, pp.118-119, 2008.

[4] Sicong You, "The Function of Web-2.0 in the Construction and Application of Distance Education Resources", Technology System, pp.66-68, 2009.

[5] Zhiyu Zhu, Jianquan Cheng, University Library Web2.0 technology Empirical Study.Modern Information, pp.- 134-137, 2009.

[6] Analysis Report for Current Situation and Profit Model of China Web2.0 from 20072008. Hui Code Market Research Report. http://www.hdemr.com/

[7] Zhang Wei, Data mining Bibliometrics,Book information, pp.57-61,2005.

[8] Jiangdong Cheng, Web2.0 Study. Ecommerce, pp.64-68,2006.

[9] Shasha Guo, Min Wei and Jiangnan Xiao, Web2.0 Research Situation at Home and Abroad and Its Implications on the Tourism Industry, Technology of Beijing International Studies University, pp.3741,2008 .

[10]Hui Ding, Web2.0 impact on enterprise information. Guide of Technology Innovation, pp.24-25,2008.

[11]Ge Wang,Web2.0 Application in Ecommerce, Information Research, pp.113115, 2008.

[12] Shaojuan Gan, The nature and application development of Web2.0, Communication hot spots, pp.6-10, 2008. 